\title{
Chikungunya Virus-Encoded nsP2, E2 and E1 Strongly Antagonize the Interferon- $\beta$ Signaling Pathway
}

\author{
Sojung Bae, Jeong Yoon Lee, and Jinjong Myoung*
}

Korea Zoonosis Research Institute, Genetic Engineering Research Institute and Department of Bioactive Material Science, Jeonbuk National University, Jeonju 561-756, Republic of Korea

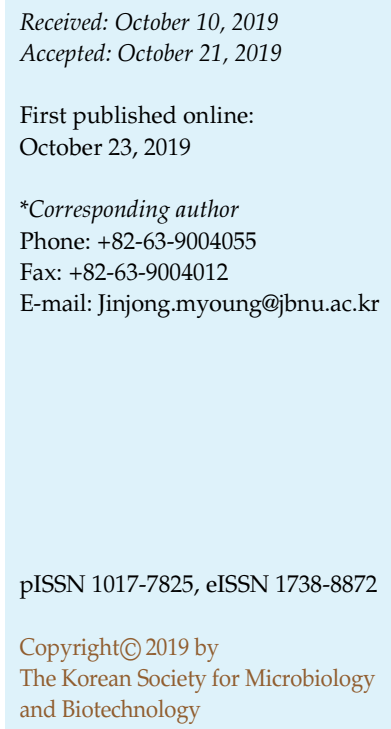

\begin{abstract}
Chikungunya virus (CHIKV) is a single-stranded positive-sense RNA virus, belonging to the genus Alphavirus of the Togaviridae family. It causes multiple symptoms, including headache, fever, severe joint and muscle pain, and arthralgia. Since CHIKV was first isolated in Tanzania in 1952, there have been multiple outbreaks of chikungunya fever. However, its pathogenesis and mechanisms of viral immune evasion have been poorly understood. In addition, the exact roles of individual CHIKV genes on the host innate immune response remain largely unknown. To investigate if CHIKV-encoded genes modulate the type I interferon (IFN) response, each and every CHIKV gene was screened for its effects on the induction of the IFN$\beta$ promoter. Here we report that CHIKV nsP2, E2 and E1 strongly suppressed activation of the IFN- $\beta$ promoter induced by the MDA5/RIG-I receptor signaling pathway, suggesting that $\mathrm{nsP2}$, E2, and E1 are the major antagonists against induction of IFN- $\beta$. Delineation of underlying mechanisms of CHIKV-mediated inhibition of the IFN- $\beta$ pathway may help develop virus-specific therapeutics and vaccines.
\end{abstract}

Keywords: Chikungunya virus, interferon, antagonist

\section{Introduction}

Chikungunya virus (CHIKV) is named after the Swahili or Makonde word Kun qunwala, meaning "bends up" [1]. CHIKV infection is associated with several illnesses such as fever, myalgia, headache, rash and joint pain [2]. Acute symptoms can last 2-3 weeks, but polyarthralgia affecting multiple joints can persist for several years [3].

CHIKV is a mosquito-borne alphavirus that was first recognized in East Africa in 1952, and primarily transmitted by Aedes aegypti and Aedes albopictus mosquitoes [1, 4]. Phylogenetically, CHIKV lineage sequences are classified into two distinct branches: West African (WA) and East/ Central/South African (ECSA) strains. The WA strain was responsible for epidemics in Africa via zoonotic transmission while those caused by the ECSA strain occur primarily in India and Southeast Asia [5]. During 2005-2006, numerous CHIKV outbreaks re-emerged in the Indian Ocean and spread to India, infecting over 1.5 million people [3]. The virus further spread to Europe and the United States via travelers [5, 6]. The latest CHIKV epidemics in Europe were reported in Italy (2007) and in France (2010) [7, 8].

CHIKV is a single-stranded positive-sense enveloped RNA virus with a diameter of $70 \mathrm{~nm}$. The $11.8 \mathrm{~kb}$ RNA genome consists of a 5'-methylguanylate cap, a 3'-polyadenylated tail and two open reading frames (ORFs). The first ORF encodes a precursor protein that is proteolytically processed into non-structural proteins; nsP1, nsP2, snP3 and nsP4 [911]. Non-structural proteins play critical roles in viral replication and transcription. The second ORF encodes six structural proteins (E3, E2, E1, C, 6K and a transframe protein [TF], the last of which is synthesized by ribosomal frameshifting) [12]. The capsid protein packages the viral RNA. E3 and $6 \mathrm{~K}$ are small proteins that function together to translocate the precursor envelope protein to the endoplasmic reticulum [13]. TF and 6K are viral accessory proteins that contribute to viral budding [14]. Glycoproteins, E1 and E2, form heterodimers to mediate the fusion of the virus and the host cell [15].

Type I interferons (IFNs) are a family of cytokines that 
induce antiviral responses and activate elements of the innate and adaptive immune systems [16-18]. Induction of type I IFN is triggered by pattern recognition receptors (PRRs) such as Toll-like receptors (TLRs) and cytosolic receptors (melanoma differentiation-associated gene 5 (MDA5) and retinoic acid-inducible gene-I (RIG-I)). Cytosolic receptors are responsible for recognition of viral doublestranded (ds) RNA, and upon cognate ligand binding, MDA5 and RIG-I mediate polymerization and activation of mitochondrial antiviral-signaling protein (MAVS) via caspase activation and recruitment domain (CARD)-CARD interactions. MAVS in turn activates TANK-binding kinase 1 (TBK1) and inhibitor of $\mathrm{\kappa B}$ kinase $\varepsilon$ (IKKE). TBK1 and IKK $\varepsilon$ phosphorylate IFN regulatory factors 3 and 7 (IRF3 and IRF7), which dimerize and translocate into the nucleus. This signaling cascade leads to production of type I IFNs through activation of the IFN- $\alpha / \beta$ promoter [19-25].

It is not well understood how CHIKV-encoded proteins interact with and modulate the MDA5/RIG-I receptor signaling pathway. We screened each and every gene of CHIKV for its capability to inhibit activation of the IFN- $\beta$ promoter. Here we report that CHIKV nsP2, E2 and E1 proteins strongly antagonize the IFN- $\beta$ signaling pathway and multiple signaling molecules seemed to be efficiently inhibited by them. Delineation of detailed mechanisms will deepen understanding of CHIKV-induced pathologies and help develop anti-CHIKV therapeutics.

\section{Material and Methods}

\section{Cells}

HEK293T cells were purchased from the American Type Culture Collection (ATCC, USA) and maintained in Dulbecco's modified Eagle's medium (Welgene, Korea) supplemented with 10\% fetal bovine serum (Welgene) and 1\% penicillin/streptomycin (Gibco, USA) at $37^{\circ} \mathrm{C}$ with $5 \% \mathrm{CO}_{2}$.

\section{Reagents}

Polyethylenimine (PEI) and complete Mini Protease Inhibitor Cocktail were purchased from Millipore Sigma (USA). The Beta-Glo Assay System and Luciferase Assay System were purchased from Promega (USA). The Opti-MEM and Pierce BCA Assay Kit were obtained from Thermo Fisher Scientific (USA). 2-Mercatoethanol and $4 \times$ Laemmli sample buffer were procured from Bio-Rad (USA). Amersham ECL western blotting detection reagent, Amersham ECL Prime western blotting detection reagent, and Amersham Protran 0.45 NC nitrocellulose western blotting membranes were purchased from GE Healthcare Life Sciences (USA). Mouse monoclonal anti-FLAG (M2) and rabbit monoclonal anti-HA were purchased from Sigma-Aldrich (USA). Rabbit anti-
GAPDH, anti-rabbit IgG and anti-mouse IgG conjugated with HRP antibodies were obtained from Cell Signaling Technology (USA). The restriction enzymes SbfI and ApaI were purchased from Enzynomics (Korea) and T4 DNA ligase was procured from New England Biolabs (USA).

\section{Virus and Viral Stocks}

A Chikungunya virus was purchased from the Korean National Culture Collection for Pathogens (accession \#43132). The virus was amplified in Vero E6 cells (ATTCC). When the genome of the CHIKV Korean isolate was fully sequenced, its genome displayed very close similarity to a Singapore strain (Pubmed accession \# FJ445484.2). CHIKV stocks were prepared and titrated on Vero E6 cells.

\section{Plasmid Construction}

Vector pcDNA3.1 (Invitrogen, USA) was used and its multiple cloning site was modified. The original vector was digested with NheI and Pmel, and ligated with a synthesized linker with a 3xFlag and spacer ( $3 x$ GGGGS). The modified vector was named pcDNA3.1Hygro-JY4-3xFLAGN-GS3 (5'-GCTAGCGCCACCATGGACTACA AGGACCACGACGGTGACTACAAGGACCACGACATCGACT ACAAGGACGACGACGACAAGCTTTCTGGTGGCGGTGGCTC GGGCGGAGGTGGGTCGGGTGGCGGCGGATCCTGCAGGCGC GCCGAATTCGAAAGCGCTATCGATATCGATGGCGCCTGGC CAGACCATCAGTCGAGTGGCGCCACTGGACTAATGGTCCG TACGCTCGACTGTACAGGCCGGCCTCAGGTTAACACCGGT ACCTCAGCCCGGGCGGCCGCATGCGGGCCCCTCGAGTCTA GAGTTTAAAC-3'). Complementary DNA was prepared from the genomic RNA of a CHIKV Korean isolate by reverse transcriptase (MMLV, Promega) according to the manufacturer's instructions. CHIKV genes were amplified by polymerase chain reaction using Pfu Plus polymerase (Elpisbio, Korea) [26]. Each gene fragment was cloned into the pcDNA3.1-Hygro-JY4-3XFlagN-GS3 vector using SbfI and ApaI restriction sites. All primers used to amplify CHIKV genes for cloning into the vectors are listed in Table 1.

\section{Luciferase and Western Blotting Assays}

HEK293T cells $\left(5 \times 10^{5}\right.$ cells/well $)$ were seeded into a 6-well plate the day before transfection. DNA-PEI complexes at a ratio of 1:2 in a total $200 \mu \mathrm{l}$ of opti-MEM (Thermo Fisher Scientific) were added onto cells dropwise; $1 \mu \mathrm{g}$ of CHIKV-encoded gene expressing plasmid, $0.5 \mu \mathrm{g}$ of plasmid encoding each signaling molecule (e.g., MDA5, RIG-I, etc), $0.5 \mu \mathrm{g}$ of IFN- $\beta$-luc and $0.1 \mu \mathrm{g}$ of $\beta$-galexpressing plasmid $[27,28]$. At $24 \mathrm{~h}$ post-transfection, cells were lysed using 1× Reporter Assay Lysis Buffer with 1× Protease Inhibitor (Millipore Sigma). The protein amount in the cell lysates was quantified using Pierce BAC Protein Assay Kit (Thermo Fisher Scientific). A $15 \mu \mathrm{g}$ protein sample was separated by $12 \%$ sodium dodecyl sulfate-polyacrylamide gel electrophoresis (SDSPAGE). The following primary antibodies were used for western blotting: mouse monoclonal anti-FLAG, rabbit monoclonal anti$\mathrm{HA}$, and rabbit anti-GAPDH antibodies. 
Table 1. Primers used to clone Chikungunya virus genes into pcDNA3.1-Hygro-JY4-3XFLAGN-GS3.

\begin{tabular}{|c|c|}
\hline Primer name & Sequence $\left(5^{\prime}-3^{\prime}\right)$ \\
\hline CHIKV-nsP1-F & GGCCTGCAGGATGGATCCTGTGTACGTGGA \\
\hline CHIKV-nsP1-R & CCG_G_C_CCTTATGCGCCCGCTCTGTCC \\
\hline CHIKV-nsP2-F & GGCCTGCূAGGGGAATAATAGAGACTCCGAGAGG \\
\hline CHIKV-nsP2-R & CCGG_G_C_CTTAGGTGACCTGTCCTACGAAG \\
\hline CHIKV-nsP3-F & GGCCTGCAGGCGAGCAGGATGTGCACCGT \\
\hline CHIKV-nsP3-R & CCG_G__C_C_TTACCTGTCTAGTCATAACTCGTCGTC \\
\hline CHIKV-nsP4-F & GGCCTEGCAGGGCAGGTGGGTATATATTCTCGTCG \\
\hline CHIKV-nsP4-R & CCGGGCCC_TTATTTAGGACCGCCGTACAAAG \\
\hline CHIKV-C-F & GGCCTGCAGGATGGAGTTCATCCCAACCCA \\
\hline CHIKV-C-R & CCG_G_C_C_TTAACTCCACTCTTCGGCCCC \\
\hline CHIKV-E3-F & GGC_TTGCAGGCTTGCCATCCCAGTTATGTGC \\
\hline CHIKV-E3-R & CCGG_G_C_CTTAACCGCCAGCGACGTAGC \\
\hline CHIKV-E2-F & GGCCTGCAGGACCAAGGACAACTTCAATGTC \\
\hline CHIKV-E2-R & CCGG__C_C_TTATTTAGCTGTTCTGATGCAGC \\
\hline CHIKV-6K-F & GGC_T_GCA_GGGCGGCCACATACCAAGAGG \\
\hline CHIKV-6K-R & CCGG_G_C_CTTAGTACGCGCTCACAGTGTGG \\
\hline CHIKV-E1-F & GGCCTGCAGGGAACACGTAACAGTGATCCCG \\
\hline CHIKV-E1-R & CCG_G__C_C_TTAGTGCCTGCTGAACGACA \\
\hline
\end{tabular}

Dotted underline: restriction site, Solid underline: CHIKV sequence.

\section{Statistical Analysis}

Two independent experiments were carried out and the data shown are the mean \pm standard deviation. For statistical analysis, a paired two-tailed Student's $t$-test was performed. The difference was considered to be statistically significant when $p<0.05$.

\section{Results}

nsP2, E2 and E1 Strongly Inhibited MDA5- and RIG-IInduced Activation of the IFN- $\beta$ Promoter

To identify the CHIKV-encoded genes involved in the modulation of the type I IFN production, CHIKV-encoded genes were cloned into an expressing vector. All genes were comparably expressed except nsP2, E1 and E2, codon optimization of which improved their protein expression levels (Fig. 1, bottom panels). As E1 and E2 form glycoprotein spikes on the viral envelope with E3 serving to chaperone the formation and maturation of E1/E2 heterodimers [29], dimers (E1E2 or E2E3) and trimers (E1E2E3) were included in the screening against MDA5- or RIG-I-mediated induction of the IFN- $\beta$ promoter (Fig. 1).

Co-expression of nsP2, E1 and E2 with MDA5 or RIG-I (Fig. 1) inhibited over $80 \%$ of both MDA5- and RIG-Imediated IFN- $\beta$ promoter activities while nsP1 displayed lower but significant inhibitory effect on that of MDA5
( 40\% inhibition). In addition, nsP2, E1 and E2 decreased protein levels of MDA5 by $40-50 \%$ (Fig. 1A, bottom panels). Furthermore, hetero-dimers and -trimers suppressed activation of the IFN- $\beta$ promoter and decreased MDA5 proteins levels as expected. Interestingly, E1, but not nsP2 and E2, down-regulated RIG-I protein levels (Fig. 1B, bottom panels) while all three proteins efficiently inhibited activation of the IFN- $\beta$ promoter (Fig. 1B, top panels). Taken together, these findings suggest that nsP2, E1 and E2 effectively suppress RIG-I-like receptor (RLR)-mediated induction of the IFN- $\beta$ promoter activities.

\section{MAVS-Mediated Induction of the IFN- $\beta$ Promoter Is} Impeded by nsP1, nsP2, E2 and E1

Next, we examined if MAVS, a downstream signaling molecule of RLRs, is regulated by CHIKV-encoded genes (Fig. 2). MAVS-mediated activation of the IFN- $\beta$ promoter was, as screened by the luciferase assay, inhibited by nsP1 (64\%), nsP2 (98\%), E2 (87\%) and E1 (97\%) (Fig. 2). Coexpression of E1, E2 and/or E3 similarly suppressed induction of the IFN- $\beta$ promoter by MAVS. In addition, nsP2 expression strongly down-regulated the protein level of MAVS while that of E1, E2, or E3, in combination or individually, had little, if any, effects on the MAVS protein levels. 

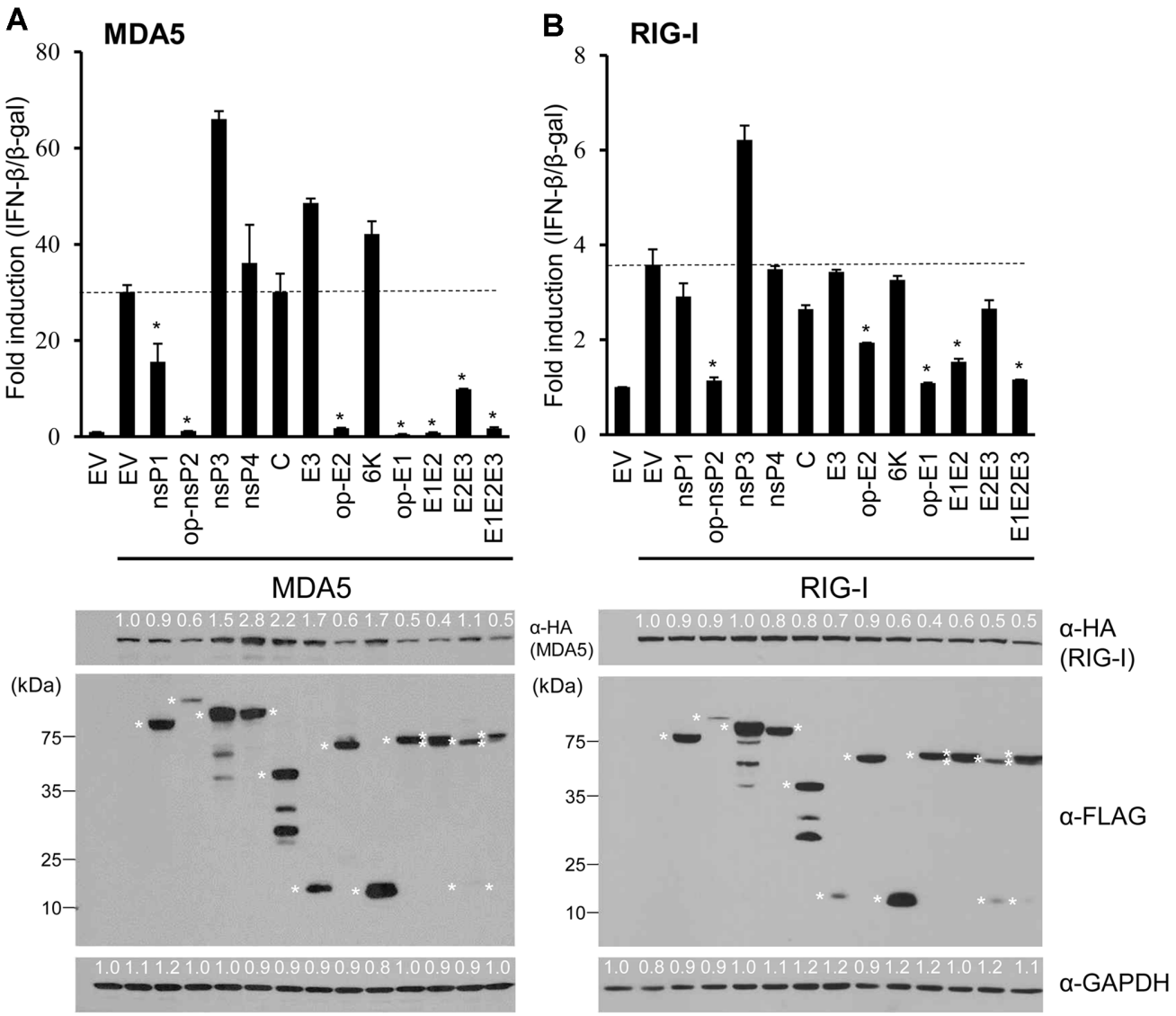

Fig. 1. Chikungunya virus (CHIKV)-encoded nsP2, E1 and E2 strongly inhibit MDA5- and RIG-I-mediated activation of the IFN- $\beta$ promoter.

HEK293T cells were co-transfected with the expression vectors for CHIKV viral genes, IFN- $\beta$-Luc, $\beta$-gal, and either MDA5 (A) or RIG-I (B). At $24 \mathrm{~h}$ post-transfection, cell lysates were prepared for luciferase assay and western blotting. Histogram plots (upper panels) represent fold induction of the IFN- $\beta$ promoter normalized to $\beta$-gal activities (means $\pm \mathrm{SD}$ ). Note that nsP2, E1 and E2 were codon-optimized as expression levels of those proteins were low to negligible. Codon-optimized genes were termed op-nsP2, op-E1 and op-E2, respectively. CHIKV proteins were blotted with $\alpha$-FLAG antibodies while MDA5 or RIG-I were with $\alpha$-HA antibodies. Asterisks indicate the size of viral genes tagged by N-terminus $3 \times$ FLAG and Spacer (3x GGGGS). MDA5 and RIG-I protein levels were analyzed by the ImageJ software and their relative expression was normalized to that of GAPDH and indicated numbers in white. The results shown represent one of two independent experiments. Statistical significance was determined by two-tailed Student's $t$-test $\left({ }^{*} p<0.05\right)$.

TBK1- and IKK $\varepsilon-M e d i a t e d$ Induction of the IFN- $\beta$ Promoter Is Inhibited by CHIKV-Encoded Genes

TBK1 and IKKE are important kinases for the phosphorylation and activation of IRF3, the key transcription factor for the induction of the IFN- $\beta$ promoter. Therefore, we tested if TBK1- and IKKE-mediated activation of the IFN- $\beta$ pathway is modulated by the co-expression of CHIKV-encoded genes. Like in the cases of RLRs and MAVS, nsP2, E1, E2 strongly antagonized TBK1- (Fig. 1A) and IKK $\varepsilon$-mediated (Fig. 1B) activation of the IFN- $\beta$ promoter by over $90 \%$ and $60 \%$, respectively. Protein levels of TBK1, when assessed by western blotting (Fig. 1A, bottom panels), were significantly down-regulated only when co-expressed with all of the glycoproteins (E1, E2, and $\mathrm{E} 3$ ). In addition, $\mathrm{nsP} 4$ and $\mathrm{C}$ were capable of antagonizing TBK1-medated induction of the IFN- $\beta$ promoter without affecting protein levels of TBK1. Interestingly, protein levels of IKK $\varepsilon$ were down-regulated to a greater extent by the expression of nsP2 (40\%), E1 (60\%) and E2 (40\%) while those of TBK1 were barely affected by the co-expression with the same proteins.

nsP2 Strongly Antagonizes Both IRF3- and IRF3-5DMediated Activation of the IFN- $\beta$ Promoter Activities

Phosphorylation of IRF3 at the C-terminus by TBK1/ IKK $\beta$ induces conformational changes, leading to IRF3 


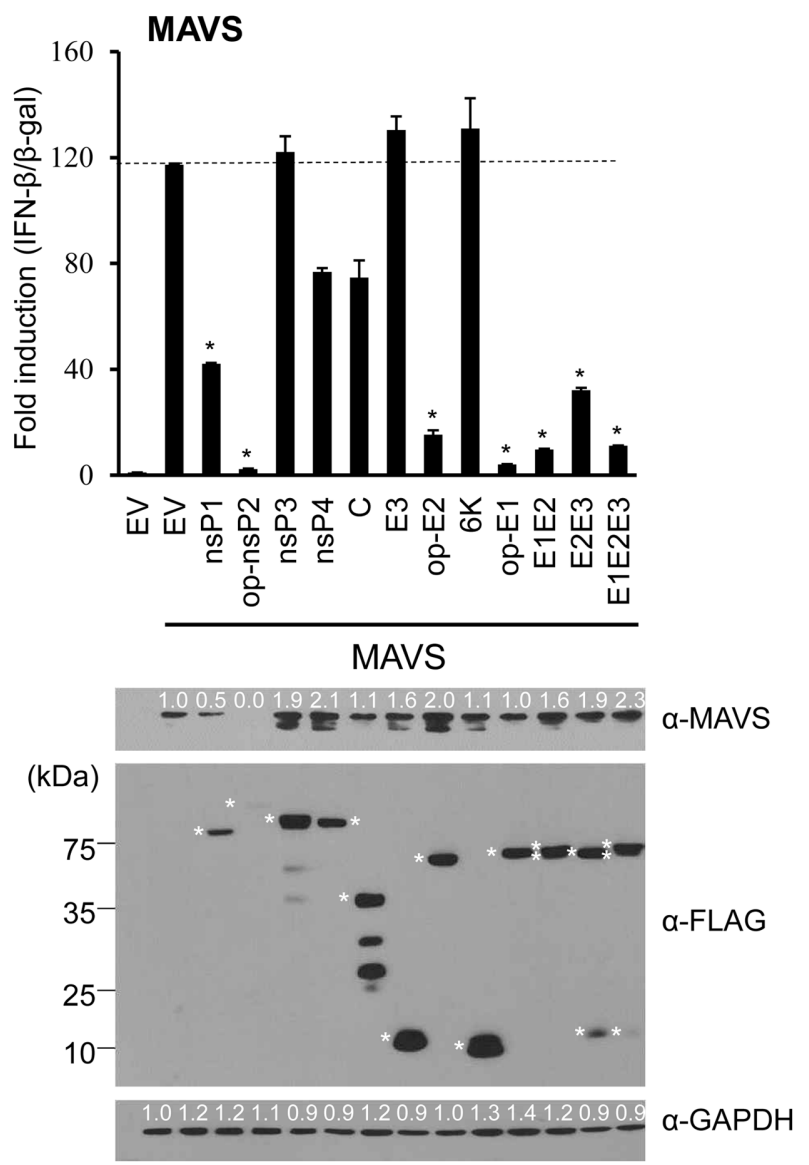

Fig. 2. nsP1, E1 and E2 impair MAVS-mediated induction of the IFN- $\beta$ promoter.

HEK293T cells were similarly transfected as described in Fig. 1. Histogram plots of fold induction of the IFN- $\beta$ (top panel) and protein levels (bottom panel), as assessed by the Firefly luciferase assay and western blotting, are shown. Statistical significance was determined by two-tailed Student's $t$-test $\left({ }^{*} p<0.05\right)$. MAVS $(59.2$ $\mathrm{kDa}$ ) was detected by anti-MAVS antibodies and its expression levels were analyzed by the ImageJ software and normalized to those of GAPDH. Data from one representative experiment of two independent experiments are presented (means $\pm \mathrm{SD}$ ).

dimerization and nuclear translocation [17, 27, 28, 30]. IRF3-5D is a constitutive active form of IRF3 which harbors 5 phosphomimetic Asp (D) substitutions of Ser (S)/Thr (T) at the C-terminus (S396D, S398D, S402D, T404D, and S405D). Thus, IRF3-5D does not require activation of upstream kinases for its activation and dimerization. E1 and nsP2 were capable of significantly down-regulating induction of IRF3-mediated activation of the IFN- $\beta$ promoter (Fig. 4A, top panel) while only nsP2 was able to suppress that of IRF-5D (Fig. 4B, top panel). On the other hand, protein levels of IRF3 or IRF3-5D were not significantly affected by the expression of nsP2 and E1 (Fig. 4, bottom panels).

\section{Discussion}

Type I IFNs are part of the innate immune response against viral infections, and in humans, IFN- $\alpha$, IFN- $\beta$, IFN$\varepsilon$, IFN- $\kappa$, and IFN- $\omega$ are known to be expressed. Once viruses infect host cells, pathogen-associated molecular patterns, such as viral glycoproteins and viral RNA, are recognized by pattern recognition receptors (PRRs), including TLRs and cytosolic RNA sensors (MDA5 and RIG-I) [31]. A cascade of signal amplification ensues, culminating in the phosphorylation and activation of IRF3 and IRF7 and their translocation into the nucleus and in the activation of the IFN- $\beta$ promoter [32]. Many viral proteins have now been known for their abilities to disrupt and alter IFN signaling. Some well-known examples include 1) the V proteins of paramyxoviruses that inhibit MDA5-mediated induction of the IFN- $\beta$ promoter [33], 2) ICP0 protein encoded by bovine herpesvirus 1 that inhibit the IFN- $\beta$ promoter activity via direct degradation of IRF3 [34], 3) HEV-encoded methyltransferase and papain-like cysteine protease that suppress MDA5- or RIG-I-mediated induction of IFN- $\beta[17,24,25,30], 4)$ ORF8b protein of the Middle East respiratory syndrome coronavirus that acts as an antagonist for activation of the IFN- $\beta$ promoter $[27,28]$.

CHIKV nsP2 is known to impair the JAK-STAT signaling by excluding STAT2 from the nucleus [35]. However, whether other CHIKV-encoded proteins contribute to the suppression of type I IFN production is unknown. Therefore, we sought to determine if CHIKV-encoded proteins are involved in the modulation of the MDA5/RIG-I receptor signaling pathway. Interestingly, CHIKV nsP2, E2 and E1 are strong antagonists against all the signaling molecules involved in the pathway; the cytosolic RNA sensors MDA5 and RIG-I (Fig. 1) and downstream signaling molecules (MAVS (Fig. 2), TBK1 and IKK $\varepsilon$ (Fig. 3)). On the other hand, only nsP2 and E1 were able to inhibit IRF3 (Fig. 4). The molecular basis of this general suppression of the IFN- $\beta$ induction pathway requires further scrutiny. A hint comes from its unique function of nsP2: host shut-off. Studies have shown that CHIKV nsP2 protein inhibits cellular transcription by inducing ubiquitination and degradation of RPB1, a catalytic subunit of the RNA polymerase II complex [36]. Thus, one may envision that the host shut-off activity of nsP2 may be responsible for down-regulation of all signaling molecules that are involved in the IFN- $\beta$ pathway. In fact, nsP2 has been known to shut off and 

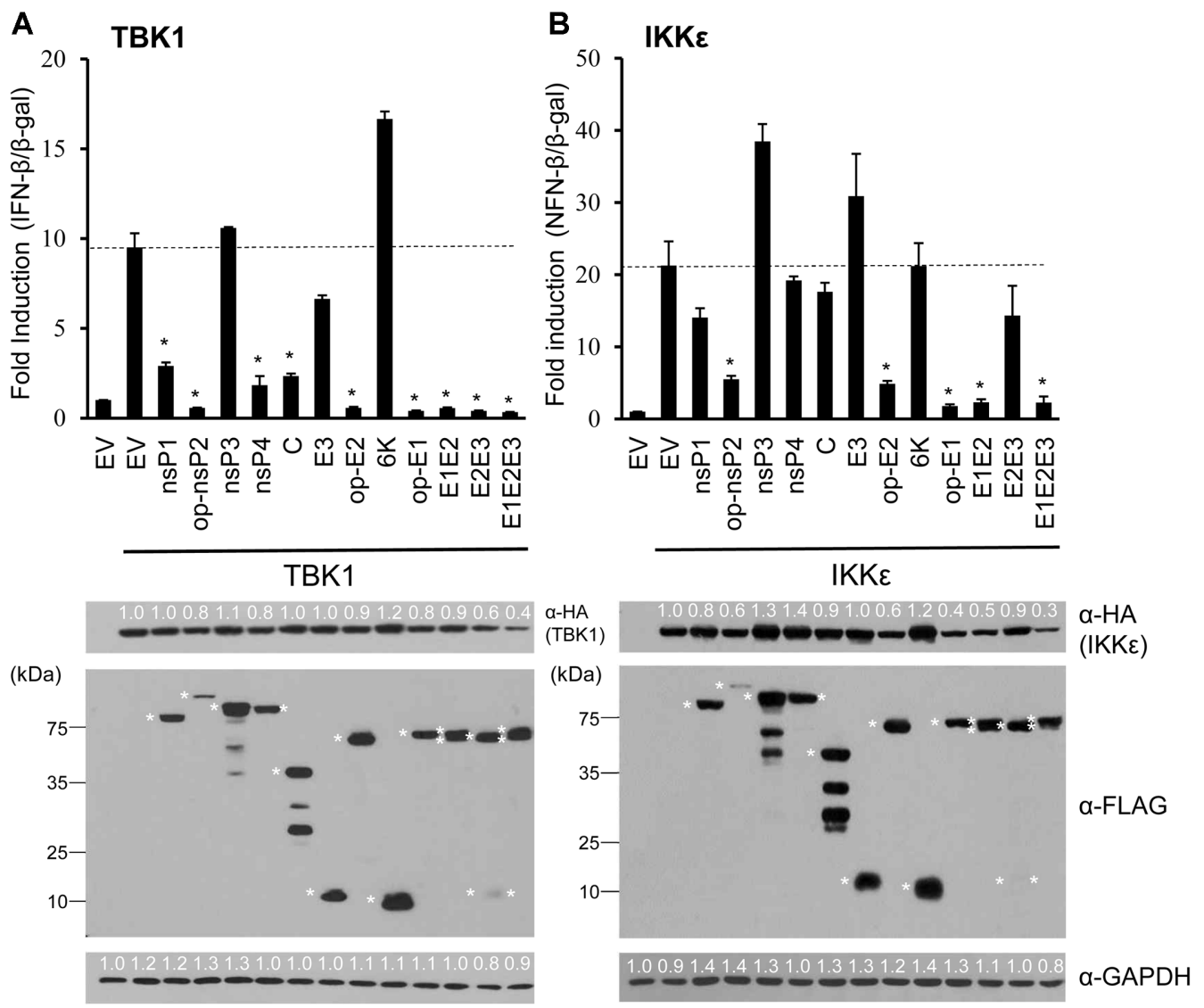

Fig. 3. Activation of the IFN- $\beta$ promoter, induced by TBK1 and IKKe, is significantly down-modulated by CHIKV-encoded proteins.

HEK293T cells were co-transfected with the expression vectors for CHIKV viral genes, IFN- $\beta$-Luc, $\beta$-gal and either TBK1 (A) or IKKE (B). At $24 \mathrm{~h}$ post-transfection, cell lysates were prepared for luciferase (upper panels) and western blotting assays (lower panels). Statistical significance was

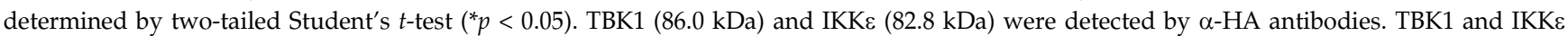
expression levels were normalized as described in Fig. 2. One representative data set of two independent experiments is shown.

suppress the host type I IFN response [37, 38]. Currently, we are testing this possibility by employing a host shut-offdeficient mutant form of nsP2. Of note, only nsP2 was able to impair IRF3-5D-mediated induction of the IFN- $\beta$ promoter without perturbation of its protein levels (Fig. 4B). Based on these data, it is tempting to postulate that E1mediated inhibition of IRF3-mediated induction of the IFN$\beta$ promoter requires inhibition of its kinases while that of nsP2 does not. It seems that nsP2-mediated inhibition may not entail its protease activity as protein levels of IRF3 and IRF3-5D remained largely unaffected (Fig. 4, bottom panels). However, we cannot formally exclude the possibility that the protease activity of nsP2 may be involved in the inhibitory effects as previous studies have shown that several RNA viral proteases inhibit the host innate immune response [39]. Furthermore, how glycoproteins, E1 and E2, confer a general suppression on the IFN signaling is totally unknown. Identification of the host proteins interacting with nsP2, E1 and E2 may shed light on the underlying mechanisms of inhibition by nsP2, E1 and E2. Of note, nsP4 and $C$ seem to specifically impair TBK1-mediated activation of the IFN- $\beta$ promoter while nsP2, E1 and E2 displayed a general inhibitory effect on the signaling molecules involved in the MDA5/RIG-I receptor signaling pathway. Further investigation is required to explain their unique contribution to the viral evasion of the type I IFN response.

In conclusion, the data presented here demonstrate that CHIKV nsP2, E1 and E2 are the major antagonists of the IFN- $\beta$ promoter activity induced by MDA5/RIG-I receptor signaling pathway. These findings improve our understanding of how CHIKV and the host interact and how CHIKV effectively evades the innate immunity. 


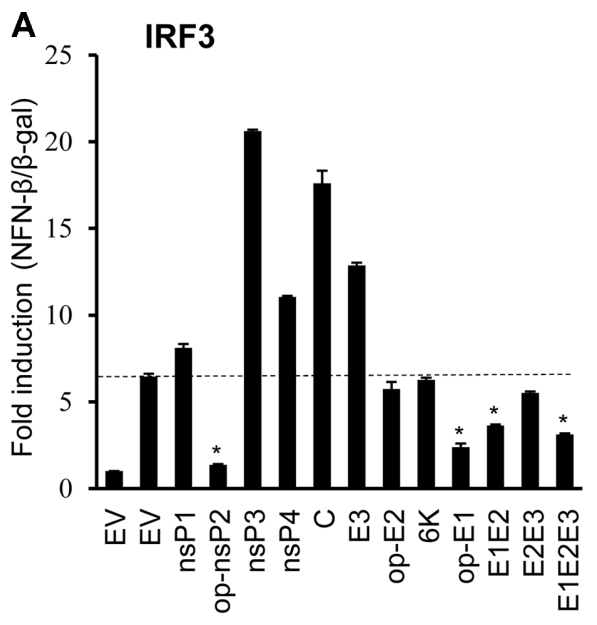

IRF3-FL

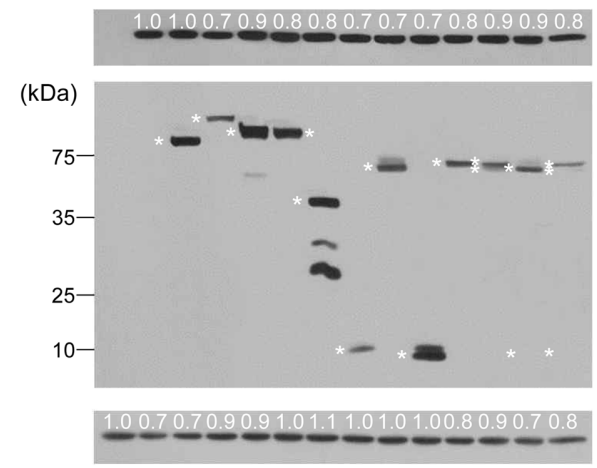

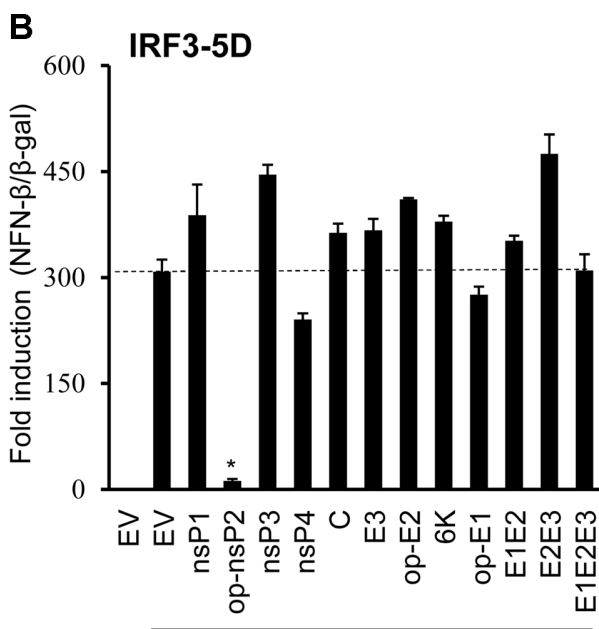

IRF3-5D

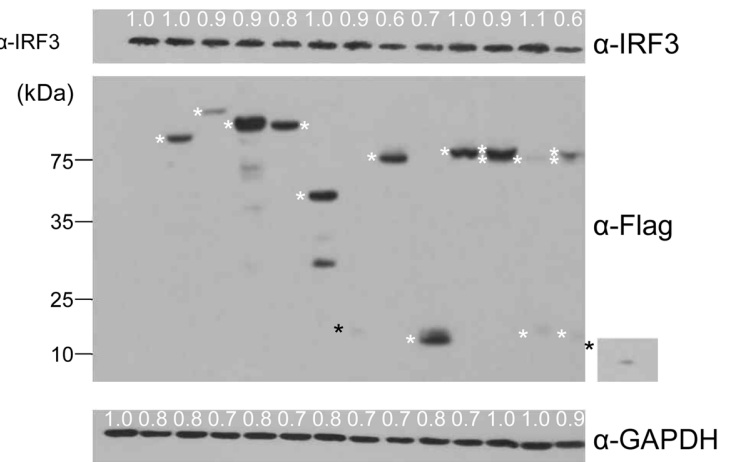

Fig. 4. nsP2 significantly impairs both IRF3- and IRF3-5D-mediated induction of the IFN- $\beta$ promoter.

HEK293T cells were co-transfected with CHIKV genes and IRF3 (A) and IRF3-5D (B). At $24 \mathrm{~h}$ post-transfection, levels of the IFN- $\beta$ promoter induction were assessed by luciferase assay (top panels). Protein expression levels were determined by western blotting (bottom panels). Statistical significance was determined by two-tailed Student's $t$-test $\left({ }^{*} p<0.05\right)$. IRF3 (50 kDa) and IRF3-5D (50.1 kDa) were detected by $\alpha$-IRF3 antibodies. IRF3 and IRF3-5D expression levels were normalized to those of GAPDH. The results shown represent one of three independent experiments (A) and one of two independent experiments (B).

\section{Acknowledgements}

This research was supported by the Basic Science Research Program of the National Research Foundation funded by the Ministry of Education (2017R1A6A1A03015876).

\section{Conflict of Interest}

The authors have no financial conflicts of interest to declare.

\section{References}

1. Robinson MC. 1955. An epidemic of virus disease in Southern Province, Tanganyika Territory, in 1952-53. I. Clinical features. Trans. R Soc. Trop. Med. Hyg. 49: 28-32.
2. Burt FJ, Rolph MS, Rulli NE, Mahalingam S, Heise MT. 2012. Chikungunya: a re-emerging virus. Lancet 379: 662-671.

3. Schwartz O, Albert ML. 2010. Biology and pathogenesis of chikungunya virus. Nat. Rev. Microbiol. 8: 491-500.

4. Manore CA, Hickmann KS, Xu S, Wearing HJ, Hyman JM. 2014. Comparing dengue and chikungunya emergence and endemic transmission in A. aegypti and A. albopictus. J. Theor. Biol. 356: 174-191.

5. Silva LA, Dermody TS. 2017. Chikungunya virus: epidemiology, replication, disease mechanisms, and prospective intervention strategies. J. Clin. Invest. 127: 737-749.

6. Gibney KB, Fischer M, Prince HE, Kramer LD, St George K, Kosoy OL, et al. 2011. Chikungunya fever in the United States: a fifteen year review of cases. Clin. Infect. Dis. 52: e121-126.

7. Rezza G, Nicoletti L, Angelini R, Romi R, Finarelli AC, Panning $M$, et al. 2007. Infection with chikungunya virus in Italy: an outbreak in a temperate region. Lancet 370: 1840-1846. 
8. Grandadam M, Caro V, Plumet S, Thiberge JM, Souares Y, Failloux AB, et al. 2011. Chikungunya virus, southeastern France. Emerg. Infect. Dis. 17: 910-913.

9. Moizeis RNC, Fernandes T, Guedes P, Pereira HWB, Lanza DCF, Azevedo JWV, et al. 2018. Chikungunya fever: a threat to global public health. Pathog. Glob. Health. 112: 182-194.

10. Law YS, Utt A, Tan YB, Zheng J, Wang S, Chen MW, et al. 2019. Structural insights into RNA recognition by the Chikungunya virus nsP2 helicase. Proc. Natl. Acad. Sci. USA 116: 9558-9567.

11. Strauss JH, Strauss EG. 1994. The alphaviruses: gene expression, replication, and evolution. Microbiol. Rev. 58: 491-562.

12. Parashar D, Cherian S. 2014. Antiviral perspectives for chikungunya virus. Biomed. Res. Int. 2014: 631642.

13. Tuekprakhon A, Puiprom O, Sasaki T, Michiels J, Bartholomeeusen K, Nakayama EE, et al. 2018. Broadspectrum monoclonal antibodies against chikungunya virus structural proteins: Promising candidates for antibody-based rapid diagnostic test development. PLoS One 13: e0208851.

14. Snyder JE, Kulcsar KA, Schultz KL, Riley CP, Neary JT, Marr S, et al. 2013. Functional characterization of the alphavirus TF protein. J. Virol. 87: 8511-8523.

15. Kielian M, Chanel-Vos C, Liao M. 2010. Alphavirus entry and membrane fusion. Viruses. 2: 796-825.

16. Stetson DB, Medzhitov R. 2006. Type I interferons in host defense. Immunity 25: 373-381.

17. Kang S, Myoung J. 2017. Host innate immunity against hepatitis E virus and viral evasion mechanisms. J. Microbiol. Biotechnol. 27: 1727-1735.

18. Kang S, Myoung J. 2017. Primary lymphocyte infection models for KSHV and its putative tumorigenesis mechanisms in B cell lymphomas. J. Microbiol. 55: 319-329.

19. Honda K, Takaoka A, Taniguchi T. 2006. Type I interferon [corrected] gene induction by the interferon regulatory factor family of transcription factors. Immunity 25: 349-360.

20. Akira S, Uematsu S, Takeuchi O. 2006. Pathogen recognition and innate immunity. Cell 124: 783-801.

21. Hu YW, Zhang J, Wu XM, Cao L, Nie P, Chang MX. 2018. TANK-binding kinase 1 (TBK1) isoforms negatively regulate Type I interferon induction by inhibiting TBK1-IRF3 interaction and IRF3 phosphorylation. Front. Immunol. 9: 84.

22. Lu LL, Puri M, Horvath CM, Sen GC. 2008. Select paramyxoviral $\mathrm{V}$ proteins inhibit IRF3 activation by acting as alternative substrates for inhibitor of kappaB kinase epsilon (IKKe)/TBK1. J. Biol. Chem. 283: 14269-14276.

23. Brisse M, Ly H. 2019. Comparative structure and function analysis of the RIG-I-Like rceptors: RIG-I and MDA5. Front. Immunol. 10: 1586.

24. Kang S, Choi C, Choi I, Han KN, Rho SW, Choi J, et al. 2018. Hepatitis E virus methyltransferase inhibits type I interferon induction by targeting RIG-I. J. Microbiol. Biotechnol. 28: $1554-1562$

25. Kim E, Myoung J. 2018. Hepatitis E virus papain-like cysteine protease inhibits type I interferon induction by down-regulating melanoma differentiation-associated gene 5 . J. Microbiol. Biotechnol. 28: 1908-1915.

26. Lee J, Bae S, Myoung J. 2019. Generation of full-length infectious cDNA clones of iddle east respiratory syndrome coronavirus. J. Microbiol. Biotechnol. 29: 999-1007.

27. Lee JY, Bae S, Myoung J. 2019. Middle East respiratory syndrome coronavirus-encoded ORF8b strongly antagonizes IFN-beta promoter activation: its implication for vaccine design. J. Microbiol. 57: 803-811.

28. Lee JY, Bae S, Myoung J. 2019. Middle east respiratory syndrome coronavirus-encoded accessory proteins impair MDA5-and TBK1-mediated activation of NF-kappaB. J. Microbiol. Biotechnol. 29: 1316-1323.

29. van Duijl-Richter MK, Hoornweg TE, Rodenhuis-Zybert IA, Smit JM. 2015. Early events in Chikungunya vrus infectionfrom virus cell binding to membrane fusion. Viruses 7: 3647-3674.

30. Myoung J, Min K. 2019. Dose-Dependent Inhibition of melanoma differentiation-associated gene 5-mediated activation of type I interferon responses by methyltransferase of hepatitis E virus. J. Microbiol. Biotechnol. 29: 1137-1143.

31. Jang JH, Shin HW, Lee JM, Lee HW, Kim EC, Park SH. 2015. An overview of pathogen recognition receptors for innate immunity in dental pulp. Mediators Inflamm. 2015: 794143.

32. Chau TL, Gioia R, Gatot JS, Patrascu F, Carpentier I, Chapelle JP, et al. 2008. Are the IKKs and IKK-related kinases TBK1 and IKK-epsilon similarly activated? Trends Biochem. Sci. 33: 171-180.

33. Andrejeva J, Childs KS, Young DF, Carlos TS, Stock N, Goodbourn S, et al. 2004. The V proteins of paramyxoviruses bind the IFN-inducible RNA helicase, mda-5, and inhibit its activation of the IFN-beta promoter. Proc. Natl. Acad. Sci. USA 101: 17264-17269.

34. Saira K, Zhou Y, Jones C. 2007. The infected cell protein 0 encoded by bovine herpesvirus 1 (bICP0) induces degradation of interferon response factor 3 and, consequently, inhibits beta interferon promoter activity. J. Virol. 81: 3077-3086.

35. Fros JJ, Liu WJ, Prow NA, Geertsema C, Ligtenberg M, Vanlandingham DL, et al. 2010. Chikungunya virus nonstructural protein 2 inhibits type I/II interferon-stimulated JAK-STAT signaling. J. Virol. 84: 10877-10887.

36. Akhrymuk I, Kulemzin SV, Frolova EI. 2012. Evasion of the innate immune response: the Old World alphavirus nsP2 protein induces rapid degradation of Rpb1, a catalytic subunit of RNA polymerase II. J. Virol. 86: 7180-7191.

37. Frolov I. 2004. Persistent infection and suppression of host response by alphaviruses. Arch. Virol. Suppl. 139-147.

38. Simmons JD, White LJ, Morrison TE, Montgomery SA, Whitmore AC, Johnston RE, et al. 2009. Venezuelan equine encephalitis virus disrupts STAT1 signaling by distinct mechanisms independent of host shutoff. J. Virol. 83: 1057110581.

39. Lei J, Hilgenfeld R. 2017. RNA-virus proteases counteracting host innate immunity. FEBS Lett. 591: 3190-3210. 